\title{
Hydrologie urbaine : modélisation et effet d'échelle
}

\author{
Urban hydrology : modelisation and scale effect
}

par B. Chocat et P. Cabane

Institut National des Sciences Appliquées de Lyon

The modelisation of the rainfall transformation on an urban catchment presents two notable particularities : $i$ ) contributing zones are relatively easily identifiable and can be interpreted as impermeable zones directely linked to the stormwater sewer network; ii) the routes followed by the water are artificial (created according to simple rules) and are easily identifiable (street and roof gutters ...). In fact, urban hydrology allows us in theory to present the processes of production and transfer in great detail, especially with the present development of geographic information systems (GIS) in numerous large cities.

The purpose of this publication is to study the repetitive nature of the modelised reaction of urban catchments when the modelisation is decreased little by little. The results of different numerical experiments will be linked with a global quantification of the geometric network.

\section{I 口 INTRODUCTION}

L'hydrologie est une science qui s'intéresse au comportement de milieux complexes, hétérogènes, soumis à des phénomènes divers interagissant les uns avec les autres. Les fondements théoriques des méthodes utilisées pour les décrire sont pour la plupart issus essentiellement de la mécanique des fluides. Ils nécessitent pour leur application des milieux homogènes et des hypothèses fortes sur la linéarité des processus. Le décalage entre les deux aspects du problème rend extrêmement difficile l'explication du comportement global d'un bassin versant par la composition de mécanismes élémentaires analysés à une échelle où le milieu est homogène. Pourtant, la curiosité scientifique continue d'entraîner de nombreux chercheurs dans une quête, peutêtre utopique, vers la mise en évidence de lois générales permettant d'expliciter rigoureusement les différents changements d'échelle nécessaires. Parmi les différents milieux étudiés, le domaine urbain est sans doute celui où cette analyse paraît a priori la plus facilement réalisable : fonctions de production simples du fait du caractère imperméable de surfaces importantes, lignes d'écoulement artificielles faciles à repérer et à décrire, grand nombre de données disponibles sur les milieux étudiés, possibilité de mesurer des flux significatifs pour de petites surfaces drainées, etc.

L'objet de cette communication n'est pas de proposer une solution au problème, mais, beaucoup plus modestement, de donner quelques pistes sur les méthodes exploitables pour progresser dans la compréhension des mécanismes de composition des fonctions de transfert. Après avoir présenté une première voie fondée sur l'expérimentation numérique montrant que les résultats obtenus à l'exutoire d'un bassin versant sont relativement insensibles à l'échelle spatiale utilisée pour le modéliser, nous proposerons quelques méthodes d'analyse plus ou moins originales, exploitant les notions de complexité et de fractalité.

\section{POSSIBILITÉS DE L'ÉTUDE DÉTAILLÉE DES BASSINS VERSANTS URBAINS}

L'étude des spécificités des bassins versants urbains au regard de leur fonctionnement hydrologique, fait apparaître quatre particularités notables :

i) les zones contributives sont relativement faciles à identifier (du moins pour les crues de faible importance) et en grande partie identifiables aux zones imperméables directement raccordées au réseau d'évacuation des eaux pluviales,

ii) l'essentiel des cheminements suivis par l'eau se fait dans des réseaux artificiels, conçus selon des règles relativement simples et générales, et également faciles à identifier (gouttières, caniveaux, réseaux de conduites d'évacuation),

iii) le fonctionnement hydraulique des ouvrages qui composent ces réseaux artificiels est le plus souvent simple, les écoulements étant généralement unidimensionnels, 
iv) le développement des systèmes d'informations géographiques (SIG) autorise une connaissance fine du terrain et des réseaux souterrains, disponible sur un support facile à utiliser. Les couches cadastrales superposées aux données structurelles du réseau d'assainissement (planimétrie, altimétrie, ouvrages spéciaux, etc.) permettent en théorie une identification spatiale fine des éléments de production et de transfert.

On peut donc en théorie décrire finement le cheminement de l'eau depuis sa tombée sur le sol jusqu'à sa collecte dans le réseau d'assainissement, puis à travers ce dernier jusqu'à l'exutoire du bassin versant étudié, en décrivant indépendamment le fonctionnement de l'ensemble des surfaces productrices et des lignes d'écoulement. En pratique, l'exercice n'est bien sûr pas aussi simple. La complexité du paysage urbain et ses multiples singularités sont autant d'obstacles à une modélisation complète des processus de production et de transfert de l'eau dans les bassins versants. Les micro-dépressions du sol, les murs de clôture, les équipements de toiture, etc., constituent des singularités très difficiles à décrire et qui jouent parfois un rôle déterminant dans le fonctionnement hydrologique d'une parcelle.

De la même façon, les réseaux d'assainissement fourmillent également de singularités qui sont autant de freins à une modélisation précise et détaillée de leur fonctionnement hydraulique. Les déversoirs d'orage, les étranglements, les canalisations d'accès, les changements de pente, de direction, etc., sont capables de modifier les écoulements de façon considérable même dans le cas de crue de faible importance.

De plus, la modélisation ne peut se faire sans garder une cohérence entre l'échelle spatiale utilisée pour décrire le milieu et celle propre aux phénomènes représentés.

$\mathrm{Si}$, en théorie, la modélisation à une échelle très fine est possible, les difficultés matérielles (temps nécessaire au recueil des informations et moyens nécessaires à leur simulation) et théoriques limitent donc le niveau de définition que l'on peut atteindre en pratique.

Il n'en demeure pas moins vrai que, même si l'on ne peut pas décomposer le système à étudier en éléments réellement homogènes vis-à-vis de leur comportement hydrologique et hydraulique, il est cependant possible de comparer les résul-

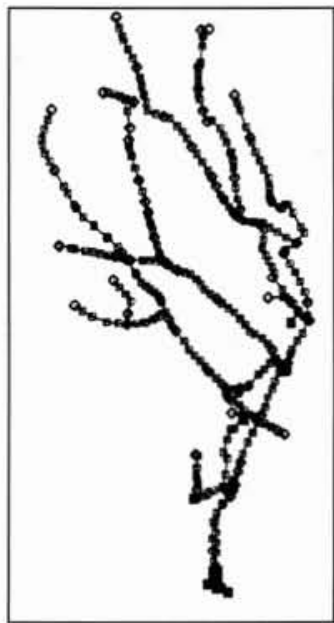

1. Plan du réseau complet.

\section{III $\square$ PRÉSENTATION DU SITE EXPÉRIMENTAL ET DE LA MÉTHODOLOGIE}

\subsection{Modélisation du bassin versant}

Le bassin étudié est drainé par un réseau d'assainissement de type unitaire possédant cinq déversoirs d'orage ne fonctionnant que lors des pluies les plus fortes. Sa structure est essentiellement ramifiée, malgré la présence d'une grande maille centrale (voir la figure 1). Il s'agit d'une zone dite périurbaine, caractérisée par la juxtaposition d'anciens villages et de zones neuves d'habitat essentiellement pavillonnaire. Le pourcentage moyen d'imperméabilisation est de l'ordre de $18 \%$. L'exutoire du bassin a été équipé d'un débitmètre de type MAINSTREAM-IRIS (capteur vitesse et sonde de pression) en décembre 1994. Les débits ont été mesurés en continu par pas de temps de 6 minutes jusqu'en janvier 1996. Les mesures vont reprendre sur ce site au printemps 1999.

Ce bassin versant a fait l'objet de cinq modélisations différentes.

La modélisation la plus fine a été conduite en conservant l'ensemble des informations disponibles dans le système GESICA. Il s'agit d'un SIG, développé par la Direction de l'eau de la Communauté urbaine de Lyon avec le soutien scientifique et technique de l'INSA. L'unité de description est le tampon (regard de visite). Toutes les conduites souterraines appartenant au réseau public (y compris les têtes de réseau) et tous les ouvrages spéciaux recensés ont été pris en compte. La longueur moyenne d'un tronçon est de 40 mètres et le réseau modélisé comporte 362 tronçons de calcul (voir figure 2). Les surfaces productrices ont été décomposées avec une précision du même ordre (70 unités hydrologiques élémentaires) en utilisant en complément à GESICA des photographies aériennes de la zone.

Dans la modélisation la plus grossière, le bassin a été représenté par une seule unité hydrologique et le réseau d'assainissement par un seul tronçon représentatif du réseau à l'exutoire, c'est-à-dire sur le point de mesure. Dans cette représentation, les cinq déversoirs d'orage existants sur le réseau ont été représentés par un seul déversoir équivalent situé à l'amont du capteur débitmétrique.

Les modèles intermédiaires correspondent à des situations

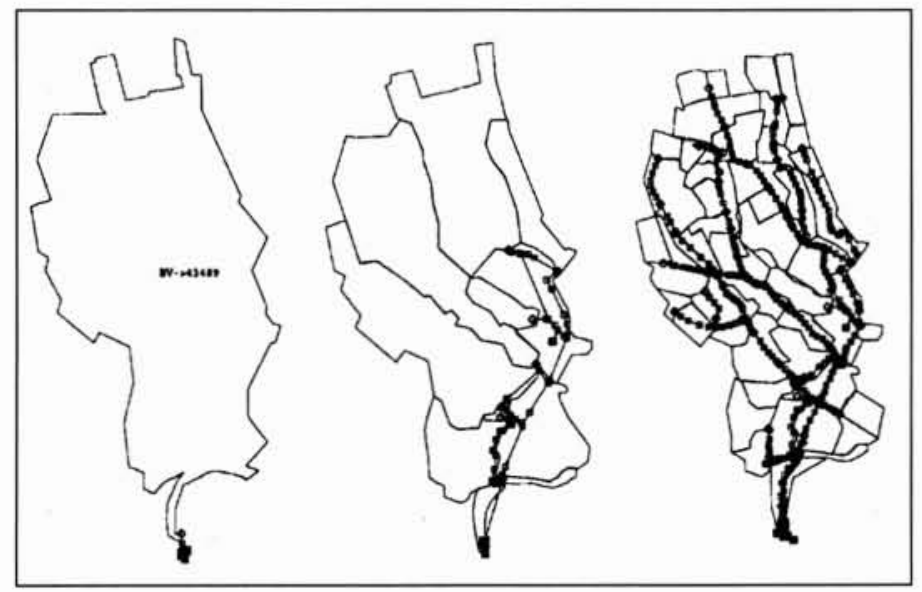

2. Plan du réseau avec 3 des 5 modèles. lisant une très grande diversité d'échelles de représentation. Le paragraphe suivant présente les résultats d'une telle étude menée sur un bassin versant urbain d'environ 250 hectares, situé à Ecully, à l'ouest de l'agglomération lyonnaise. Ce bassin déjà suivi lors d'une campagne de mesures en 1995 sera instrumenté dans le cadre d'un observatoire de terrain en hydrologie urbaine (OTHU) en cours de développement sur la communauté urbaine de Lyon. 
dégradées de la première, telles que le bassin versant et le réseau d'assainissement soient décrits de moins en moins précisément dans l'espace. Ils comportent respectivement 30 unités hydrologiques élémentaires et 80 tronçons, puis 15 unités et 30 tronçons, enfin 8 unités et 10 tronçons.

La réduction du nombre d'éléments (éléments du réseau ou unités hydrologiques) ne s'est pas faite selon des règles rigides et automatiques comme celles présentées dans l'étude de Eberl et al [1], mais a été effectuée de façon "intelligente" afin de ne pas altérer la réalité du fonctionnement du réseau.

Ainsi certaines parties importantes du réseau du point de vue hydraulique ont été conservées (zone amont et aval des déversoirs d'orage) pour les projets 3 et 4 .

\subsection{Modèles de simulation utilisés et méthodologie}

Les simulations ont été effectuées avec logiciel de simulation CANOE développé par notre laboratoire en relation avec la société SOGREAH [2].

La partie production a été modélisée par une perte initiale et un coefficient de ruissellement moyen par unités hydrologiques et par gamme d'événements pluvieux. Un calage effectué sur les volumes à l'exutoire a montré que ce type de fonction de production très sommaire représentait assez bien le comportement des surfaces contributives pour une grande gamme d'événements pluvieux (même s'il ne couvre probablement pas l'ensemble des crues de la normale à l'extrême !).

La partie transfert sur les bassins versants a été représentée par le modèle du réservoir linéaire, le "lag time", paramètre unique du modèle étant estimé de façon prévisionnelle (sans calage) par un ajustement proposé par Desbordes [3].
Le modèle hydraulique utilise les équations complètes de Barré Saint Venant ; la mise en charge et les débordements du réseau sont pris en compte grâce à l'artifice de la fente de Preissman et une cote de débordement (cote du sol) pour chaque nœud modélisé. Les déversoirs d'orage sont représentés par des lois de seuils [4].

Chacun des cinq modèles du réseau a été simulé pour différentes précipitations réelles, représentatives des pluies les plus fortes observées au cours d'une année sur la région lyonnaise (pluies de type frontal, orageux). Les simulations ont été effectuées en conservant des valeurs identiques pour tous les paramètres (pas de temps calcul en particulier).

Pour chacune de ces pluies (qui mettent toutes en charge le réseau de façon partielle ou totale !), nous avons comparé les hydrogrammes à l'exutoire fournis par les différents modèles avec ceux mesurés. Cinq critères d'écart ont été utilisés : écart relatif sur le débit de pointe, écart relatif sur le volume, écart quadratique normé pondéré on non, critère de Nash.

\section{IV — RÉSULTATS OBTENUS ET INTERPRÉTATION}

Les figures 3 et 4 illustrent les résultats obtenus.

Comme l'illustrent ces figures, la simplification du modèle ne conduit pas nécessairement à une dégradation des résultats. Les écarts observés doivent en effet être rapportés à la valeur des incertitudes sur les débits mesurés, évalués à $\pm 30 \%$ par Musso [5].

Dans tous les cas, comme l'illustre la figure 3, les hydrogrammes obtenus sont très voisins dans les phases de montée

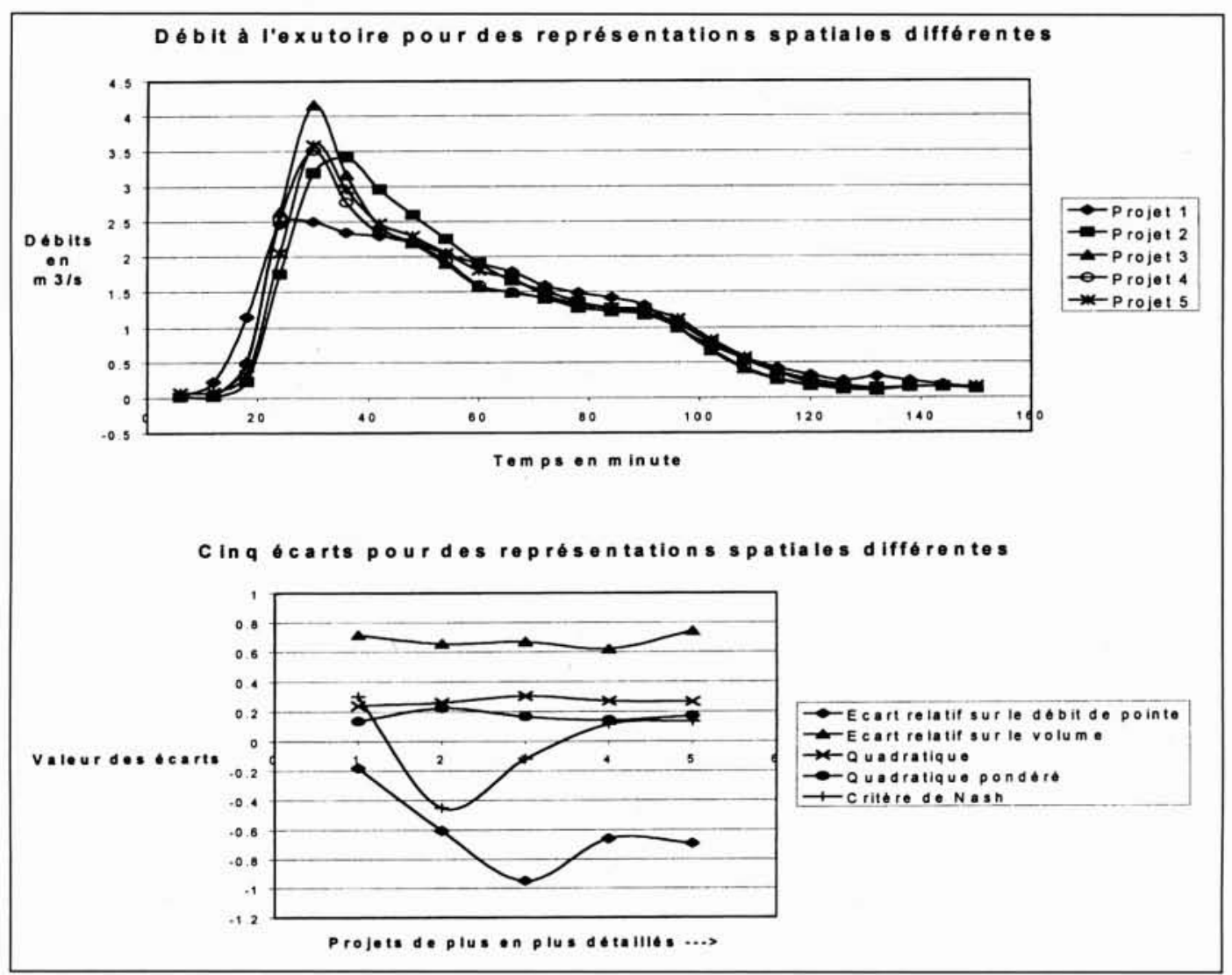

3 et $4:$ Exemples de résultats. 
de la crue et de la décrue. Les différences sont plus importantes lorsque le débit est fort. Ce phénomène est essentiellement dû au fonctionnement des déversoirs d'orage. La réduction du nombre d'éléments du réseau affecte en effet la précision avec laquelle ces ouvrages spéciaux sont pris en compte. Par exemple, le modèle le plus dégradé ne possède qu'un seul déversoir représentatif des cinq autres. La définition d'un déversoir unique équivalent à cinq déversoirs situés dans des lieux différents s'avère très difficile.

En revanche, l'écart entre le projet 4 et le projet 5 est très faible. L'effort de réduction entre les deux projets a été réalisé de façon non uniforme dans l'espace, c'est-à-dire que la modélisation est restée aussi fine dans les deux projets aux endroits les plus sensibles du réseau (en particulier au voisinage des déversoirs d'orage) et dégradée largement par ailleurs. Cela montre qu'une description adaptée à l'échelle des phénomènes donne d'aussi bons résultats qu'une description détaillée.

Ces résultats recoupent ceux de Eberl et al [1] ou de Blanpain et al [6]. Les écarts entre les résultats sont très faibles même avec une réduction considérable du nombre d'éléments composant la modélisation du bassin versant. Par exemple, les écarts quadratique ou quadratique pondéré restent globalement constants lors de la dégradation de la finesse de représentation spatiale du bassin versant.

Le remplacement d'une fonction hydraulique détaillée, appliquée sur un système complexe et en tenant compte de la distribution réelle des zones de production par une fonction de transfert extrêmement simple de type hydrologique (réservoir linéaire) ne semble donc avoir que peu d'influence sur la forme des hydrogrammes à l'exutoire.

Il est alors légitime de s'interroger sur les raisons qui permettent à un modèle linéaire à un seul paramètre de représenter de façon aussi correcte une multitude de phénomènes non linéaires se déroulant dans un système extrêmement compliqué.

\section{$V$ DISTRIBUTION DES SURFACES PRO- DUCTRICES ET ANALYSE FRACTALE}

Lorsque l'on est confronté au problème précédent, une démarche classique consiste à faire appel à la théorie de la complexité [7]. D'après cette théorie, un système constitué d'un grand nombre d'éléments en interactions (ce qui est notre cas) aurait tendance à s'auto-organiser de façon à produire des formes simples ou à générer des comportements simples. L'idée de base que l'on cherche généralement à exploiter est que les surfaces produisant le ruissellement ne sont pas distribuées au hasard sur le bassin versant. L'organisation sous-jacente régularisant les signaux de crue serait ainsi due à la régularité de cette distribution.

Cette idée est ancienne, même si son expression a évolué au cours des 20 dernières années. Par exemple, la méthode des courbes isochrones repose sur l'étude de la distribution des surfaces productives en fonction de leur distance à l'exutoire. De la même façon, des classifications comme celle de Stralher [8] ressortent également de la morphométrie et visent à qualifier de façon simple le système complexe qu'est le réseau.

La notion de dimension fractale a redonné une seconde jeunesse à ce type d'approche. Cette méthode consiste à analyser les grandeurs représentatives d'un système en supposant que leur mesure s'exprime en fonction d'une puissance non entière de la grandeur fournie par l'instrument de mesure. Mandelbrot, qui a imaginé cette notion, donnait l'exemple de la mesure d'une cote dont la dimension est en fait intermédiaire entre une longueur et une surface. Dès 1976, Réméniéras [9] notait "(la notion de dimension fractale) est sans doute susceptible d'asseoir la modélisation des bassins versants sur des paramètres dont la signification physique sera indiscutable". Depuis, de nombreux auteurs ont essayé avec plus ou moins de bonheur d'utiliser cette idée (voir par exemple le numéro spécial de Journal of Hydrology de décembre 1996 [10]).

Dans le domaine de l'hydrologie urbaine, Thibault [11], en étudiant le réseau d'assainissement de l'agglomération lyonnaise a ainsi montré que le développement spatial des réseaux d'assainissement à partir de l'exutoire pouvait se mettre sous la forme :

$$
C(u)=a \cdot u^{D}
$$

Avec $u$ : rayon d'un cercle centré sur l'exutoire,

$C(u)$ : longueur totale du réseau contenu dans le cercle de rayon $u$.

Le paramètre $D$, compris entre 1 et 2 , représente la dimension spatiale du réseau. Sa valeur dépend du degré de développement du réseau et de la topographie. Moyennant des hypothèses simples (proportionnalité entre la surface drainée et la longueur de réseau drainante et vitesse d'écoulement sensiblement constante), cette approche permet d'établir une relation très significative entre la morphologie du réseau et son fonctionnement. Elle explique en particulier pourquoi le modèle du réservoir linéaire s'applique très bien aux bassins versants urbains.

Malgré ces percées intéressantes, ces travaux mériteraient d'être approfondis : prise en compte du réseau de surface, prise en compte de discontinuités dans le développement du réseau, établissement de relations déterministes ou statistiques entre $a$ et $D$ d'une part, et les paramètres de différents modèles de transfert d'autre part, etc.

\section{VI $\square$ ESPACE DES PHASES ET ATTRAC- TEURS ÉTRANGES}

\subsection{Définition}

Pour notre part, nous avons préféré défricher une autre piste, plus originale en hydrologie, même si certains auteurs l'ont déjà envisagée [12]. Il s'agit également d'une technique simple d'analyse des systèmes complexes qui consiste à étudier la forme du signal de sortie dans l'espace des phases [7].

Sur un plan pratique, le modèle à un seul paramètre du réservoir linéaire permet de bien représenter la réponse du bassin versant. Cela nous permet de conjecturer que l'espace des phases est de dimension 2 (un seul facteur explicatif). Nous traçons donc la relation $Q s(t+D t)=\mathrm{f}(Q s(t))$ (débit de sortie au temps $t+D t$ en fonction du débit de sortie au temps $t$ ). Si l'hypothèse de complexité est vraie, cette relation devrait être caractérisée par un attracteur étrange unique caractéristique du bassin versant. 


\subsection{Représentation des mesures dans l'espace des phases}

La figure 5 ci-dessous représente cette relation dans le cas des mesures effectuées pour différentes crues pour le bassin versant d'Ecully.

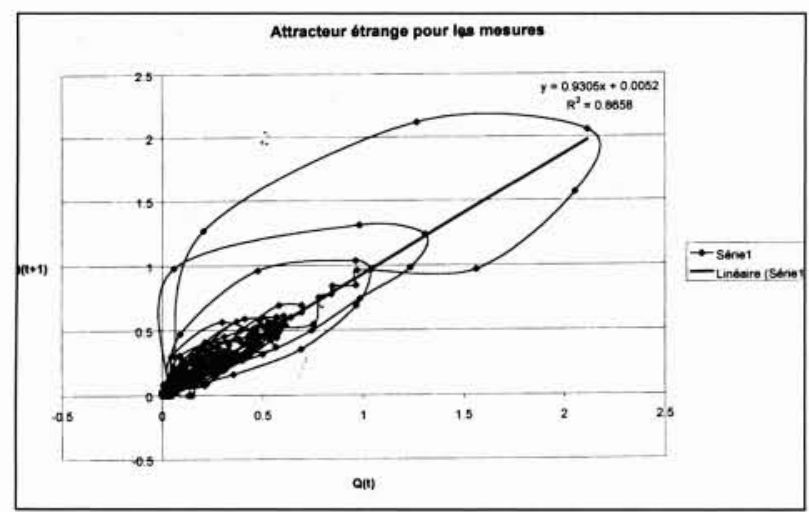

5. Attracteur étrange.

\section{3 - Interprétation}

Ces figures 6 et 7 montrent que l'ensemble des courbes s'inscrit dans une figure simple qui peut être considérée comme l'attracteur représentatif du bassin versant.

Pour une crue simple (un seul maximum), on obtient une courbe de la forme présentée en figure 6 . Une courbe de ce type peut s'interpréter de la façon suivante :

- Le rayon $\mathrm{R}$ du cercle dans lequel la fonction est inscrite représente le débit maximum. Dans le cas d'un réseau d'assainissement équipé de déversoirs d'orage, sa valeur est essentiellement dépendante du filtrage réalisé par ces déversoirs.

- La pente de la droite $\mathrm{AB}$ autour de laquelle s'organisent les points lors de la décrue est représentative du temps de réponse du bassin versant. On peut noter que le modèle du réservoir linéaire s'écrit sous la forme :

$$
Q s(t+D t)=\mathrm{e}^{-D t / K} Q s(t)+\left(1-\mathrm{e}^{-D t / K}\right) Q e(t+D t)
$$

Lors de la décrue, le débit d'entrée $Q e$ est nul. La relation entre le débit sortant et le débit entrant est donc bien linéaire, la pente de la droite étant égale à $\mathrm{e}^{-D t / K}$. Cette valeur est bornée par 1 en valeur supérieure (pas de décrue significative, le débit reste constant).

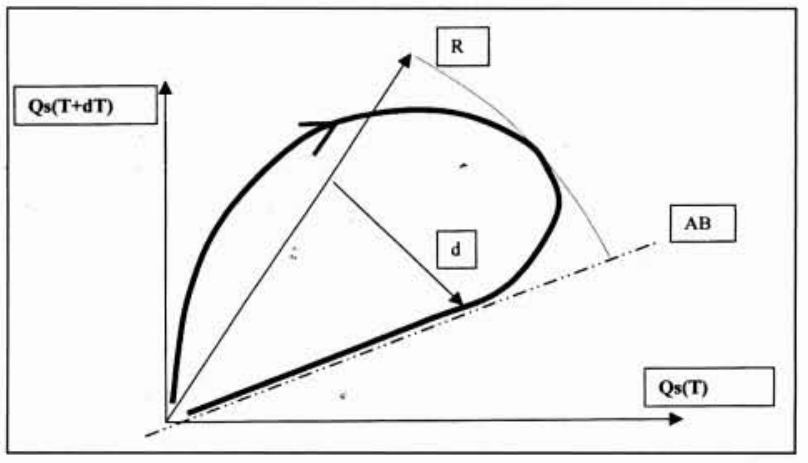

6. Attracteur étrange.
- L'ouverture $(d)$ dépend à la fois de la pluie (plus la pluie est intense et plus $d$ augmente), de la fonction de production et également du temps de réponse. En reprenant la formule du réservoir linéaire et en supposant que $Q e$ est constant, l'ouverture est alors égale au produit de la quantité (1- $\left.\mathrm{e}^{-D t / K}\right)$ $Q e$ par le cosinus de l'angle que fait la courbe de décrue avec l'axe des x (Rappelons que la tangente de cet angle est $\left.\mathrm{e}^{-D t / K}\right)$ (voir la figure 7).

\section{VII — REPRÉSENTATION DES RÉSULTATS DE SIMULATION DANS L'ESPACE DES PHASES}

Sur la figure 8 , nous pouvons voir que la finesse de la représentation n'influe pas sur la montée de la crue ni sur la décrue. La décrue se fait selon une droite caractéristique. Ici, l'équation de la droite caractéristique de la décrue est : $Q s(t+D t)=0,9259 Q s(t)+0,0401$.

\section{VIII $\square$ CONCLUSIONS}

Les trois approches présentées ici sont complémentaires.

L'expérimentation numérique montre que des modèles simples représentant globalement une succession de nombreux phénomènes microscopiques complexes peuvent donner de très bons résultats. Le bassin versant urbain et son réseau d'évacuation des eaux se comportent de façon macroscopique, dans le cas étudié, comme un filtre. La réponse à une entrée très variable semble présenter des caractéristiques relativement stables, susceptibles d'être expliquées par un seul paramètre caractéristique du bassin versant.

Ce constat laisse supposer que le comportement physique d'un bassin versant urbain soumis à une pluie pourrait être régi par des règles simples.

L'hypothèse que ces règles soient dues à une régularité dans la disposition des surfaces productrices peut être testée en utilisant la notion de dimension fractale.

Plus généralement une analyse du signal de sortie dans l'espace des phases peut permettre d'identifier différentes grandeurs caractéristiques du bassin versant et/ou de la plu-

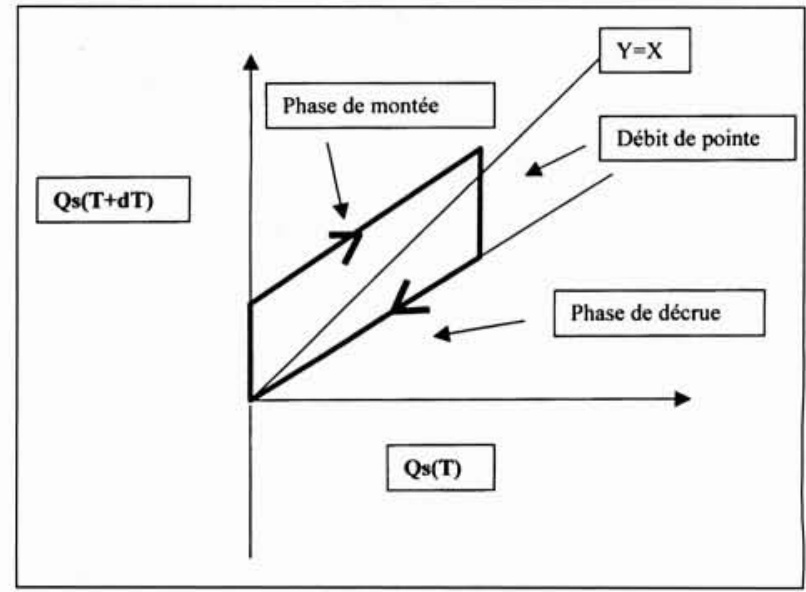

7. Forme de la relation $Q s(t+D t)=f(Q s(t))$ dans le cas du modèle du réservoir linéaire et d'un signal d'entrée $Q(e)=Q(e) d(t),(d(t)$ fonction de Dirac). 


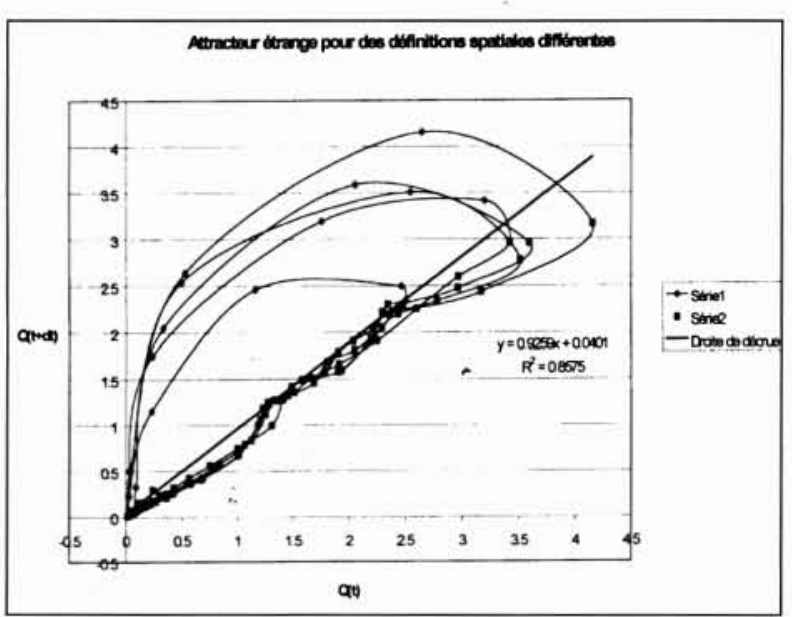

8. Attracteur étrange.

viométrie. Sur le cas simple étudié, cette analyse permet de comprendre pourquoi la représentation du bassin versant par une seule unité hydrologique simulée par le modèle du réservoir linéaire donne de bons résultats.

Le travail présenté ici est bien évidemment uniquement exploratoire. La méthodologie utilisée, reposant sur l'étude d'un seul site avec un nombre restreint de crues, devra largement être étendue pour pouvoir évaluer la généralité des résultats obtenus.

\section{BIBLIOGRAPHIE}

[1] H.EBERL. A.KHELIL, P.WILDERER : "Construction of simplified descriptions of a sewer system to characterize its hydraulic behaviour" : ICUSD 96 : Hannover : pp. 1569-1574: 1996.

[2] B.CHOCAT : "Un nouveau logiciel français d'aide à la conception et à l'exploitation des systèmes d'assainissement : NOE" : revue Génie urbain : pp. $43-48$; novembre 1997.

[3] M.DESBORDES : "Réflexions sur les méthodes de calcul des réseaux urbains d'assainissement" ; thèse Docteur ingénieur ; Université des Sciences et Techniques du Languedoc ; Montpellier ; 171 p. ; 1974.

[4] INSA/SOGREAH : "Notice du logiciel CANOE" : INSA de Lyon ; à paraître.

[5] E.MUSSO : "Evaluation des rejets de temps de pluie de grands bassins versants urbains : contribution méthodologique à la comparaison des outils et à l'estimation des incertitudes " ; thèse Docteur ingénieur : INSA Lyon ; Lyon : 363 p : 1997.

[6] L.PETIT, O.BLANPAIN, J.AL-HAJJAR : "Etude de sensibilité d'un modèle d'écoulement à la qualité des données : application aux réseaux d'assainissement pluvial" ; Novatech 98 ; pp. 247-254; 1998.

[7] I.STEWART : "Dieu joue-t-il aux dés ?" ; Paris ; Flammarion : $441 \mathrm{p} ; 1992$.

[8] A.N.STRAHLER : "Quantitative Analysis of watershed geomorphology" ; Trans. Am. Geophy. Union ; vol 38, n6 ; pp. 913-920 ; 1957.

[9] G.REMENIERIAS : "L'hydrologie de l'ingénieur" ; Paris, Eyrolles ; 251 p. : 1976.

[10] JOURNAL OF HYDROLOGY : Special issue : Fractals, scaling and nonlinear variability in hydrology ; Vol $187 ; 1996$.

[11] S.THIBAULT : "Modélisation morphofonctionnelle des réseaux d'assainissement à l'aide du concept de dimension fractale" : Thèse Docteur d'état ; INSA. Lyon ; France ; 303 p. ; 1987.

[12] V.KLEMES : "Conceptualisation and scale in hydrology" ; Journal of hydrology : pp.1-22; 1983 . 